\title{
Generalized Fractional Programming and Cutting Plane Algorithms ${ }^{1}$
}

\author{
A. I, Barros ${ }^{2}$ and J. B. G. Frenk ${ }^{3}$
}

Communicated by S. Schaible

\begin{abstract}
In this paper, we introduce a variant of a cutting plane algorithm and show that this algorithm reduces to the well-known Dinkelbach-type procedure of Crouzeix, Ferland, and Schaible if the optimization problem is a generalized fractional program. By this observation, an easy geometrical interpretation of one of the most important algorithms in generalized fractional programming is obtained. Moreover, it is shown that the convergence of the Dinkelbach-type procedure is a direct consequence of the properties of this cutting plane method. Finally, a class of generalized fractional programs is considered where the standard positivity assumption on the denominators of the ratios of the objective function has to be imposed explicitly. It is also shown that, when using a Dinkelbach-type approach for this class of programs, the constraints ensuring the positivity on the denominators can be dropped.
\end{abstract}

Key Words. Generalized fractional programming, boundedly lower subdifferentiable functions, cutting plane algorithms.

\section{Introduction}

Let $\mathscr{X} \subseteq \mathbb{R}^{n}$ be a compact set, and let $w_{i}: \mathscr{S} \rightarrow \mathbb{R}^{p}, i \in I:=\{1, \ldots, m\}$, $m \geq 1$, be a set of continuous functions on the open set $\mathscr{S}$ with $\mathscr{X} \subseteq \mathscr{S}$ and with range $\mathscr{K}_{i}$. If $f: \mathscr{K} \rightarrow \mathbb{R}$ with $U_{i \in I} \mathscr{K}_{i} \subseteq \mathscr{K} \subseteq \mathbb{R}^{p}$ denotes a boundedly lower subdifferentiable function on $\mathscr{K}$ with blsd bound $N$ and if $h: \mathscr{X} \rightarrow \mathbb{R}$

\footnotetext{
${ }^{1}$ The authors like to thank the anonymous referees and Frank Plastria for their constructive remarks on an earlier version of this paper.

${ }^{2}$ Assistant Lecturer, DEIO, Universidade de Lisboa, Lisboa, Portugal. This research was carried out at Erasmus University, Rotterdam, The Netherlands and was supported by JNICT, Lisboa, Portugal, under Contract BD/707/90-RM.

${ }^{3}$ Associate Professor, Econometric Institute, Erasmus University, Rotterdam, The Netherlands.
} 
is given by

$$
h(x):=\max _{i \in I} f\left(w_{i}(x)\right),
$$

consider the optimization problem

(P) $\inf _{x \in \mathscr{X}} h(x)$.

To solve the optimization problem $(\mathrm{P})$, a cutting plane method is introduced. This method is a generalization of a procedure discussed by Plastria (Ref. 1). It is shown in Section 3 that this method reduces to the Dinkelbachtype algorithm for a generalized fractional programming problem (Ref. 2), and so this yields a geometrical and at the same time classical interpretation of one of the most important and seemingly ad hoc algorithms in generalized fractional programming. Moreover, by this interpretation, we obtain immediately for a generalized fractional programming problem an upper and a lower bound on the optimal objective value, and so by comparing these bounds an objective-value stopping rule can be constructed. This result is not clear immediately from the original Dinkelbach-type algorithm. Finally, a class of generalized fractional programs not satisfying the standard positivity assumption of the denominator of the objective function is considered. For this class of optimization problems, it is shown that each member of this class can be handled by a Dinkelbach-type algorithm applied to a corresponding standard generalized fractional program. Moreover, an example of such a problem occurring in location theory is outlined.

\section{Cutting Plane Algorithm}

Before discussing a general cutting plane method to solve optimization problem (P), we need to introduce the following notation (Ref. 1). For an arbitrary function $f: \mathscr{K} \rightarrow \mathbb{R}$ with domain $\mathscr{K} \subseteq \mathbb{R}^{n}$, its strict lower level set of level $\mu$ is given by

$$
\mathscr{L}_{f}(\mu):=\{y \in \mathscr{K} \mid f(y)<\mu\} .
$$

Moreover, the function $f: \mathscr{K} \rightarrow \mathbb{R}$ is called lower subdifferentiable at $z \in \mathscr{K}$ if there exists some $z^{*} \in \mathbb{R}^{n}$ satisfying

$$
f(y) \geq f(z)+\left\langle y-z, z^{*}\right\rangle,
$$

for every $y$ belonging to $\mathscr{L}_{f}(f(z))$, with $\langle\cdot, \cdot\rangle$ denoting the inner product. The set of these so-called lower subgradients $z^{*}$ is denoted by $\partial^{-} f(z)$. The function $f$ is called lower subdifferentiable on $\mathscr{K}$ if the set $\partial^{-} f(z)$ is nonempty for every $z$ belonging to $\mathscr{K}$. Observe that $\partial^{-} f(z)$ equals $\mathbb{R}^{n}$ if $z$ is a minimal 
point of $f$ on $\mathscr{K}$. Finally, the function $f$ is called boundedly lower subdifferentiable (blsd) on $\mathscr{K}$ with blsd bound $N$ if $f$ is lower subdifferentiable on $\mathscr{K}$ and, for each $z \in \mathscr{K}$, there exists a lower subgradient $z^{*} \in \partial^{-} f(z)$ with Euclidean norm $\left\|z^{*}\right\|$ bounded from above by $N$. The above generalization of convexity was first studied by Plastria (Refs. 1 and 3 ) and independently by Konnov (Ref. 4). Moreover, its relation to the generalized conjugation theory of Moreau was discussed by Martínez-Legaz (Ref. 5). Among other properties, it was proved by Plastria (Ref. 1) that every blsd function $f$ is Lipschitz continuous on $\mathscr{K}$. This implies, by the continuity of the functions $w_{i}: \mathscr{S} \rightarrow \mathbb{R}^{p}, i \in I$, and by the compactness of $\mathscr{X}$ that the optimization problem $(\mathrm{P})$ is solvable; i.e., there exists some $x \in \mathscr{X}$ solving problem (P). Moreover, if $x_{k} \in \mathscr{X}$ is a nonoptimal point, the set

$$
\mathscr{L}_{h}\left(h\left(x_{k}\right)\right)=\left\{x \in \mathscr{X} \mid h(x)<h\left(x_{k}\right)\right\}
$$

is nonempty and equals

$$
\bigcap_{i \in I}\left\{x \in \mathscr{X} \mid f\left(w_{i}(x)\right)<f\left(w_{i_{k}}\left(x_{k}\right)\right)\right\},
$$

with $i_{k}$ belonging to the set $I\left(x_{k}\right)$ of active indices at $x_{k}$; i.e.,

$$
I\left(x_{k}\right):=\left\{i \in I \mid f\left(w_{i}\left(x_{k}\right)\right)=h\left(x_{k}\right)\right\} .
$$

Since $f: \mathscr{K} \rightarrow \mathbb{R}$ is by assumption boundedly lower subdifferentiable with blsd bound $N$, we obtain for every $i \in I, \alpha_{i_{k}} \in \partial^{-} f\left(w_{i_{k}}\left(x_{k}\right)\right)$, and $x \in \mathscr{L}_{h}\left(h\left(x_{k}\right)\right)$ that

$$
\begin{aligned}
f\left(w_{i}(x)\right) & \geq f\left(w_{i_{k}}\left(x_{k}\right)\right)+\left\langle w_{i}(x)-w_{i_{k}}\left(x_{k}\right), \alpha_{i_{k}}\right\rangle \\
& =h\left(x_{k}\right)+\left\langle w_{i}(x)-w_{i_{k}}\left(x_{k}\right), \alpha_{i_{k}}\right\rangle .
\end{aligned}
$$

Hence, it follows that, for every $x \in \mathscr{L}_{h}\left(h\left(x_{k}\right)\right)$,

$$
h(x) \geq h_{k}(x):=h\left(x_{k}\right)+\max _{i \in I}\left\langle w_{i}(x)-w_{i_{k}}\left(x_{k}\right), \alpha_{i_{k}}\right\rangle .
$$

Clearly the function $h_{k}: \mathscr{X} \rightarrow \mathbb{R}$, depending uniquely on the index $i_{k} \in I\left(x_{k}\right)$ and the lower subgradient $\alpha_{i_{k}} \in \partial^{-} f\left(w_{i_{k}}\left(x_{k}\right)\right)$, is properly defined; but only for $x_{k} \in \mathscr{X}$ nonoptimal is it a lower bound of $h$ on $\mathscr{L}_{h}\left(h\left(x_{k}\right)\right)$.

By (1), it is possible to introduce iteratively a set of subproblems $\left(P_{j+1}\right)$, $j \geq 0$, for the solution of (P). Let $x_{k} \in \mathscr{X}, 0 \leq k \leq j$, be given with $x_{k}, 1 \leq k \leq j$, an optimal solution of $\left(\mathrm{P}_{k}\right)$; let $x_{0}$ be an initial feasible solution; and construct for each $x_{k}$ a continuous function $h_{k}: \mathscr{X} \rightarrow \mathbb{R}$ given by (1). The subproblem $\left(\mathrm{P}_{j+1}\right)$ has now the following form:

$$
\left(\mathbf{P}_{j+1}\right) \min _{x \in \mathscr{X}} \max _{0 \leq k \leq j} h_{k}(x)
$$


Denoting by $\vartheta(\mathrm{P})$ [respectively, $\vartheta\left(\mathrm{P}_{j+1}\right)$ ] the optimal objective value of problem (P) [respectively, $\left(\mathbf{P}_{j+1}\right)$ ], the proposed cutting plane method is described as follows.

\section{Cutting Plane Algorithm}

Step 1. Choose $x_{0} \in \mathscr{X}$. Set $U B \leftarrow+\infty$, and set $j \leftarrow 0$.

Step 2. Take any $i_{j} \in I\left(x_{j}\right)$ and $\alpha_{i j} \in \partial^{-} f\left(w_{i j},\left(x_{j}\right)\right)$ with $\left\|\alpha_{i_{j}}\right\| \leq N$. If $U B>h\left(x_{j}\right)$, then set $U B \leftarrow h\left(x_{j}\right)$ and set $\bar{x} \leftarrow x_{j}$.

Step 3. If $\left\|\alpha_{i j}\right\|=0$, then stop. Otherwise, set $j \leftarrow j+1$ and solve problem $\left(\mathrm{P}_{j}\right)$.

Step 4. If $\vartheta\left(\mathrm{P}_{j}\right)<U B$, then let $x_{j}$ be an optimal solution of problem $\left(\mathrm{P}_{j}\right)$, and return to Step 2. Otherwise, stop.

The above problem (P) might have local optimal solutions. Also, since we do not assume besides continuity any additional structure on the functions $w_{i}, i \in I$, function $h$ might not be boundedly subdifferentiable, and so the usual cutting plane approach (first-order approximation around $x$ ) cannot solve these optimization problems. However, the above procedure, by locally approximating $h$ by a first-order approximation around $w_{i_{k}}\left(x_{k}\right)$ instead of $x_{k}$, will discover for these optimization problems a global solution. At the same time, the algorithm yields during its execution a lower and an upper bound for the optimal objective value. Clearly, the efficiency and implementation of this procedure depends on the structure of the subproblems. Moreover, this approach, based on classical principles, gives a clear interpretation to the seemingly ad hoc Dinkelbach procedure. Finally, if each $w_{i}$ is the identity mapping and the set $I$ consists of one element, the cutting plane method of Plastria is recovered (Ref. 1).

To analyze the properties of the above conceptual algorithm, denote by $j^{*}$ the number of times the main step was started by the algorithm. Clearly, if $j^{*}$ equals $+\infty$, the algorithm does not stop, while for $j^{*}$ finite either $\vartheta\left(\mathrm{P}_{j^{*}}\right) \geq U B$ or $\left\|\alpha_{i^{*}}\right\|$ equals zero. The proof of the next result is related to the proof of Lemma 2.1 in Ref. 6.

Proposition 2.1. The sequence $\vartheta\left(\mathrm{P}_{j}\right), j<j^{*}$, is nondecreasing and $\vartheta\left(\mathbf{P}_{j}\right) \leq \vartheta(\mathbf{P})$, for every $j<j^{*}$. Moreover, if $j^{*}$ is finite, then $\bar{x}$ is an optimal solution of $(\mathbf{P})$.

Proof. Clearly, by the definition of $\left(\mathrm{P}_{j}\right)$, it follows that

$$
\vartheta\left(\mathbf{P}_{j}\right) \leq \vartheta\left(\mathbf{P}_{j+1}\right), \quad \text { for every } 1 \leq j<j^{*} .
$$


If $j<j^{*}$ and $x_{0}, \ldots, x_{j-1}$ contain an optimal solution of (P), then at the $j$ th iteration the upper bound $U B$ equals $\vartheta(\mathrm{P})$. This implies, since $j<j^{*}$ and hence $\vartheta\left(\mathbf{P}_{j}\right)<U B$, that $\vartheta\left(\mathbf{P}_{j}\right)<\vartheta(\mathbf{P})$. Moreover, if the sequence $x_{0}, \ldots, x_{j-1}$ contains no optimal solution of $(\mathrm{P})$, then the set

$$
\mathscr{L}_{j-1}:=\bigcap_{k=0}^{j-1} \mathscr{L}_{h}\left(h\left(x_{k}\right)\right)
$$

is nonempty, and so by (1) we obtain

$$
\vartheta\left(\mathrm{P}_{j}\right) \leq \min _{x \in \mathscr{L}_{j-1}} \max _{0 \leq k \leq j-1} h_{k}(x) \leq \min _{x \in \mathscr{L}_{j-1}} h(x)=\vartheta(\mathrm{P})
$$

Also, if $j^{*}$ is finite and the algorithm stops due to $\left\|\alpha_{i^{*}}\right\|=0$, it follows by the definition of a lower subgradient that $\bar{x}=x_{j^{*}}$ is an optimal solution of (P). On the other hand, if the algorithm stops due to $\vartheta\left(\mathrm{P}_{j^{*}}\right) \geq U B$ and $\bar{x}$ is nonoptimal, we obtain that

$$
U B=h(\bar{x})=\min _{0 \leq k \leq j^{*}-1} h\left(x_{k}\right)>\vartheta(\mathrm{P}) .
$$

By (2), it follows that $\vartheta\left(\mathrm{P}_{j^{*}}\right) \leq \vartheta(\mathrm{P})$, and so $\vartheta\left(\mathrm{P}_{j^{*}}\right)<U B$, which contradicts the stopping rule.

In the cutting plane method, it may happen that some $x_{j}$ is an optimal solution of $(\mathrm{P})$, but this is not detected immediately due to $\vartheta\left(\mathrm{P}_{j+1}\right)<\vartheta(\mathrm{P})$. If the algorithm performs an infinite number of iterations (i.e., $j^{*}=+\infty$ ), one can prove the following result.

Proposition 2.2. If $j^{*}$ equals $+\infty$, every accumulation point of the sequence $\left\{x_{j}\right\}_{j \geq 0}$ is an optimal solution of (P). Moreover, the sequence $\left\{\vartheta\left(\mathrm{P}_{j}\right)\right\}_{j \geq 1}$ converges from below to $\vartheta(\mathrm{P})$.

Proof. For $\mathscr{X} \subseteq \mathbb{R}^{n}$ compact, it follows that the sequence $\left\{x_{j}, j \geq 0\right\} \subseteq \mathscr{X}$ has accumulation points in $\mathscr{X}$. Let $x_{\infty}$ be such a point (i.e., there exists a subsequence $J \subseteq \mathbb{N}$ with $\lim _{j \in J \rightarrow \infty} x_{j}=x_{\infty}$ ), and suppose by contradiction that $h\left(x_{\infty}\right)>\vartheta(\mathrm{P})+\epsilon_{0}$ for some $\epsilon_{0}>0$. Since $\lim _{j \in J \rightarrow \infty} x_{j}=x_{\infty}$, we can find, by the continuity of $h$ and $w_{i}, i \in I$, and by $f$ boundedly lower subdifferentiable with blsd bound $N$, some $l_{0} \in J$ satisfying $h\left(x_{n}\right)>\vartheta(\mathrm{P})+\epsilon_{0}$ and

$$
\left\langle w_{i_{l_{0}}}\left(x_{n}\right)-w_{i_{t_{0}}}\left(x_{l_{0}}\right), \alpha_{i_{l_{0}}}\right\rangle \leq N \max _{i \in I}\left(\left\|w_{i}\left(x_{n}\right)-w_{i}\left(x_{l_{0}}\right)\right\|\right) \leq \epsilon_{0} / 2,
$$

for every $i_{l_{0}} \in I\left(x_{l_{0}}\right)$ and $n \geq l_{0}, n \in J$. Hence by (3), it follows that

$$
h\left(x_{l_{0}}\right)+\left\langle w_{i_{0}}\left(x_{n}\right)-w_{i_{0}}\left(x_{l_{0}}\right), \alpha_{i_{i_{0}}}\right\rangle \geq h\left(x_{l_{0}}\right)-\epsilon_{0}>\vartheta(\mathrm{P}) .
$$


However, by Proposition 2.1, we obtain for $n \geq l_{0}, n \in J$ that

$$
\vartheta(\mathrm{P}) \geq \vartheta\left(\mathrm{P}_{n}\right) \geq h\left(x_{l_{0}}\right)+\left\langle w_{i_{l_{0}}}\left(x_{n}\right)-w_{i_{l_{0}}}\left(x_{l_{0}}\right), \alpha_{i_{l_{0}}}\right\rangle,
$$

and this contradicts (4), implying that $x_{\infty}$ is an optimal solution of (P).

To prove that

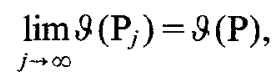

we observe that, for any $l \in J, i_{l} \in I\left(x_{l}\right)$, and $n>l, n \in J$,

$$
\begin{aligned}
0 \leq \Re(\mathrm{P})-\vartheta\left(\mathrm{P}_{n}\right) & \leq \Re(\mathrm{P})-h\left(x_{i}\right)-\left\langle w_{i_{l}}\left(x_{n}\right)-w_{i_{l}}\left(x_{i}\right), \alpha_{i_{i}}\right\rangle \\
& \leq-\left\langle w_{i_{l}}\left(x_{n}\right)-w_{i_{l}}\left(x_{l}\right), \alpha_{i_{l}}\right\rangle .
\end{aligned}
$$

As in (3), the last expression can be made arbitrarily small, and applying the monotonicity of $\vartheta\left(\mathbf{P}_{j}\right), j \geq 1$, yields the desired result.

Note that, for a large class of blsd functions, it is possible to construct a lower subgradient (Refs. 1 and 6-8), and so in principle it is possible to implement the above algorithm for this class. Finally, observe that the convergence proof requires explictly that all previous generated cuts $x \mapsto h_{k}(x)$ should be retained.

\section{Generalized Fractional Programming}

Before discussing the relation between the cutting plane method and the Dinkelbach-type algorithm, we analyze for $\mathscr{K}:=\left[\delta_{1}, \Delta_{1}\right] \times\left[\delta_{2}, \Delta_{2}\right]$, $\delta_{2}>0$, the function $f: \mathscr{K} \rightarrow \mathbb{R}$ given by $f(z):=z_{1} / z_{2}$. This function is both quasiconvex and quasiconcave and is differentiable on $\mathscr{K}$. Moreover, it is easy to check that

$$
\langle y-z, \nabla f(z)\rangle=\left(y_{2} / z_{2}\right)[f(y)-f(z)],
$$

for all $z, y \in \mathscr{K}$. By this relation, it follows that $f$ is boundedly subdifferentiable on $\mathscr{K}$. Also for

$$
\mathscr{L}_{f}(f(z)):=\{y \in \mathscr{K} \mid f(y)<f(z)\}
$$

nonempty, we obtain by (6) that

$$
\left\{\lambda \nabla f(z) \mid \lambda \geq z_{2} / \delta(z)\right\} \subseteq \partial^{-} f(z),
$$

with

$$
\delta(z):=\inf \left\{y_{2} \mid y \in \mathscr{L}_{f}(f(z))\right\} \geq \delta_{2},
$$


while for $\mathscr{L}_{f}(f(z))$ empty, it follows by definition that

$$
\partial^{-} f(z) \equiv \mathbb{R}^{2}
$$

By (7), it is clear that in general a lower subgradient depends on the domain $\mathscr{K}$.

Although not necessary for the verification of the equivalence between the cutting plane method and the Dinkelbach-type algorithm of Crouzeix, Ferland, and Schaible (Ref. 2), it is interesting to give a complete characterization of $\partial^{-} f(z)$. Since the set $\mathscr{L}_{f}(f(z))$ might be empty at the boundary of $\mathscr{K}$, we assume for simplicity that $z \in \operatorname{int}(\mathscr{K})$. If this holds, it is easy to verify that $\mathscr{L}_{f}(f(z))$ is nonempty. Moreover, since $\nabla f(z) \neq 0$ for every $z \in \mathscr{K}$, it follows that the open halfspace

$$
\mathscr{H}^{-}(\nabla f(z)):=\left\{d \in \mathbb{R}^{2} \mid\langle d, \nabla f(z)\rangle<0\right\}
$$

is always nonempty and its closure equals

$$
\left\{d \in \mathbb{R}^{2} \mid\langle d, \nabla f(z)\rangle \leq 0\right\} .
$$

For $z \in \operatorname{int}(\mathscr{K})$ we obtain now, for every $d \in \mathscr{H}^{-}(\nabla f(z))$, that

$$
z+t d \in \mathscr{L}_{f}(f(z))
$$

with $t>0$ sufficiently small, and so

$$
\mathscr{H}^{-}(\nabla f(z)) \subseteq \bigcup_{\lambda>0} \lambda\left(\mathscr{L}_{f}(f(z))-\{z\}\right) .
$$

The other inclusion can be proved using (6), and hence this yields

$$
\mathscr{H}^{-}(\nabla f(z))=\bigcup_{\lambda>0} \lambda\left(\mathscr{L}_{f}(f(z))-\{z\}\right) .
$$

The above relation is in general not true for $z$ belonging to the boundary of $\mathscr{K}$. Since $\nabla f(z) \neq 0$, and hence $z$ belongs to the closure $\operatorname{cl}\left(\mathscr{L}_{f}(f(z))\right)$ of the nonempty set $\mathscr{L}_{f}(f(z))$, we can introduce the normal cone $\mathscr{N}(z)$ of $\mathscr{L}_{f}(f(z))$ at $z \in \operatorname{int}(\mathscr{K})$, i.e.,

$$
\mathcal{N}(z):=\left\{z^{*} \in \mathbb{R}^{2}:\left\langle y-z, z^{*}\right\rangle \leq 0, \text { for every } y \in \mathscr{L}_{f}(f(z))\right\} .
$$

It is now possible to give a complete characterization $\partial^{-} f(z)$ for $z \in \operatorname{int}(\mathscr{K})$.

Lemma 3.1. If $f: \mathscr{K} \rightarrow \mathbb{R}$, with $\mathscr{K}:=\left[\delta_{1}, \Delta_{1}\right] \times\left[\delta_{2}, \Delta_{2}\right], \delta_{2}>0$, is given by $f(z)=z_{1} / z_{2}$, then for every $z \in \operatorname{int}(\mathscr{K})$, it follows that

$$
\partial^{-} f(z)=\left\{\lambda \nabla f(z) \mid \lambda \geq z_{2} / \delta(z)\right\},
$$


with

$$
\delta(z):=\inf \left\{y_{2} \mid y \in \mathscr{L}_{f}(f(z))\right\}
$$
that

Proof. By (7), we have only to verify $\subseteq$. To prove this, we first show

$$
\partial^{-} f(z) \subseteq \mathscr{N}(z), \quad \text { for any } z \in \operatorname{int}(\mathscr{K}) .
$$

Let $z^{*} \in \partial^{-} f(z)$, and consider an arbitrary $y \in \mathscr{L}_{f}(f(z))$. If $\left\langle y-z, z^{*}\right\rangle>0$, we obtain that

$$
f(y) \geq f(z)+\left\langle y-z, z^{*}\right\rangle>f(z),
$$

and so $y$ does not belong to $\mathscr{L}_{f}(f(z))$. This yields a contradiction, and hence $z^{*} \in \mathcal{N}(z)$.

To derive an alternative description of $\mathscr{N}(z), z \in \operatorname{int}(\mathscr{K})$, we observe by (9) that

$$
\begin{aligned}
\mathscr{N}(z) & =\left\{z^{*} \in \mathbb{R}^{2} \mid\left\langle d, z^{*}\right\rangle \leq 0, \text { for every } d \in \mathscr{H}^{-}(\nabla f(z))\right\} \\
& =\left\{z^{*} \in \mathbb{R}^{2} \mid\left\langle d, z^{*}\right\rangle \leq 0, \text { for every } d \in \operatorname{cl}\left(\mathscr{H}^{-}(\nabla f(z))\right)\right\} \\
& =:\left(\operatorname{cl}\left(\mathscr{H}^{-}(\nabla f(z))\right)\right)^{0},
\end{aligned}
$$

with $\left(\operatorname{cl}\left(\mathscr{H}^{-}(\nabla f(z))\right)\right)^{0}$ the polar of the closed half-space $\operatorname{cl}\left(\mathscr{H}^{-}(\nabla f(z))\right)$. Since this last set equals

$$
\left\{d \in \mathbb{R}^{2} \mid\langle d, \nabla f(z)\rangle \leq 0\right\},
$$

it is the polar of the closed set

$$
\mathscr{B}:=\{\lambda \nabla f(z) \mid \lambda \geq 0\}
$$

Applying now Theorem 14.1 of Ref. 9 yields by (10) and the above observation that

$$
\mathscr{N}(z)=\left(\mathscr{B}^{0}\right)^{0}=\mathscr{B},
$$

and we have shown that

$$
\partial^{-} f(z) \subseteq\{\lambda \nabla f(z) \mid \lambda \geq 0\}
$$

If $z^{*} \in \partial^{-} f(z)$, it follows by the above inclusion that there exists some $\lambda \geq 0$ such that

$$
f(y)-f(z) \geq \lambda\langle y-z, \nabla f(z)\rangle,
$$

for any $y \in \mathscr{L}_{f}(f(z))$. By (6), this implies

$$
\left(z_{2} / y_{2}\right)\langle y-z, \nabla f(z)\rangle \geq \lambda\langle y-z, \nabla f(z)\rangle
$$


for every $y \in \mathscr{L}_{f}(f(z))$, and since again by (6) it follows that

$$
\langle y-z, \nabla f(z)\rangle<0,
$$

we obtain that

$$
\lambda \geq z_{2} / \delta(z)
$$

The above result improves, for this special case, a more general result on the lower subgradient set for the ratio of a convex and a concave function given by Proposition 2.1 of Ref. 8 .

Returning to our algorithm, we will show that the cutting plane method discussed in Section 2 reduces to the Dinkelbach algorithm (Ref. 10) for classical fractional programming $(m=1)$ and to the Dinkelbach-type algorithm of Crouzeix, Ferland, and Schaible (Ref. 2) for generalized fractional programming $(m>1)$. For this optimization problem, it follows that $w_{i}: \mathscr{S} \rightarrow \mathbb{R}^{2}$ is given by

$$
w_{i}(x):=\left[u_{i}(x), v_{i}(x)\right],
$$

with $v_{i}(x)>0$ for every $x \in \mathscr{X}$. Moreover, the ratio function $f(z)=z_{1} / z_{2}$ is defined on $\mathscr{K}=\left[\delta_{1}, \Delta_{1}\right] \times\left[\delta_{2}, \Delta_{2}\right]$ satisfying

$$
\begin{aligned}
& \delta_{1}:=\min _{x \in \mathscr{X}} \min _{i \in I} u_{i}(x) \leq \max _{x \in \mathscr{X}} \max _{i \in I} u_{i}(x)=: \Delta_{1}, \\
& 0<\delta_{2}:=\min _{x \in \mathscr{X}} \min _{i \in I} v_{i}(x) \leq \max _{x \in \mathscr{X}} \max _{i \in I} v_{i}(x)=: \Delta_{2} .
\end{aligned}
$$

Finally, we obtain that

$$
h(x)=\max _{i \in I}\left\{u_{i}(x) / v_{i}(x)\right\} .
$$

Since $w_{i_{k}}\left(x_{k}\right)$ belongs to $\mathscr{K}$, it is clear by (7) and (8) that a lower subgradient $\alpha_{i_{k}} \in \partial^{-} f\left(w_{i_{k}}\left(x_{k}\right)\right)$ is given by

$$
\alpha_{i_{k}}:=v_{i_{k}}\left(x_{k}\right) \nabla f\left(w_{i_{k}}\left(x_{k}\right)\right) / \delta_{2}=\left(1 / \delta_{2}\right)\left[1,-h\left(x_{k}\right)\right]^{\top} .
$$

Notice that $\alpha_{i_{k}}$ is never zero. Moreover by (6), we obtain

$$
\begin{aligned}
\left.\left\langle w_{i}(x)-w_{i_{k}}\left(x_{k}\right), \alpha_{i_{k}}\right), \alpha_{i_{k}}\right\rangle & =\left[v_{i}(x) / \delta_{2}\right]\left[u_{i}(x) / v_{i}(x)-u_{i_{k}}\left(x_{k}\right) / v_{i_{k}}\left(x_{k}\right)\right] \\
& =\left(1 / \delta_{2}\right)\left[u_{i}(x)-h\left(x_{k}\right) v_{i}(x)\right],
\end{aligned}
$$

and so the function $h_{k}: \mathscr{X} \rightarrow \mathbb{R}$ given by (1) reduces to

$$
h_{k}(x)=h\left(x_{k}\right)+\left(1 / \delta_{2}\right) \max _{i \in I}\left\{u_{i}(x)-h\left(x_{k}\right) v_{i}(x)\right\} .
$$

We are now able to prove the following result. 
Lemma 3.2. If the standard assumptions for a generalized fractional program are satisfied and the cutting plane method applied to this special case stops after a finite number of steps, then $\bar{x}$ equals $x_{j^{*}-1}$, and this is the first optimal solution among $x_{0}, \ldots, x_{j^{*}-1}$. Moreover, the sequence $h\left(x_{j}\right)$, $0 \leq j<j^{*} \leq+\infty$, is strictly decreasing, and for every $x \in \mathscr{X}$ it follows that $\max _{0 \leq k \leq j} h_{k}(x)=h_{j}(x)$.

Proof. Since $\alpha_{i_{k}}, k=0,1, \ldots$, is never zero, the algorithm can stop only if $\vartheta\left(\mathrm{P}_{j}\right) \geq U B$. Therefore, if it stops after a finite number of steps, it follows by the last part of Proposition 2.1 that there exists an optimal solution of (P) among $x_{0}, \ldots, x_{j^{*}-1}$. Suppose now that $x_{k}$ with $k<j^{*}-1$ is the first optimal solution of (P) among $x_{0}, \ldots, x_{j^{*}-1}$. Hence by (11), we obtain that

$$
\vartheta\left(\mathrm{P}_{k+1}\right) \geq h_{k}\left(x_{k+1}\right) \geq h\left(x_{k}\right)+\left(1 / \delta_{2}\right)\left\{u_{i_{k+1}}\left(x_{k+1}\right)-h\left(x_{k}\right) v_{i_{k+1}}\left(x_{k+1}\right)\right\} .
$$

Since $x_{k}$ is an optimal solution,

$$
u_{i_{k+1}}\left(x_{k+1}\right)-h\left(x_{k}\right) v_{i_{k+1}}\left(x_{k+1}\right) \geq 0,
$$

and thus $\vartheta\left(P_{k+1}\right) \geq h\left(x_{k}\right)$. By this inequality and $U B=h\left(x_{k}\right)$ at the beginning of the $(k+1)$ th main step, the algorithm should have stopped at step $k+1<j^{*}$, and so $x_{0}, \ldots, x_{j^{*}-2}$ are not optimal. By the first part, this implies that $x_{j^{*}-1}$ is optimal. that

Also by (11) and Proposition 2.1, we obtain for every $1<j<j^{*} \leq \infty$

$$
\vartheta(\mathrm{P}) \geq \vartheta\left(\mathrm{P}_{j}\right) \geq h_{j-1}\left(x_{j}\right) \geq h\left(x_{j-1}\right)+\left(1 / \delta_{2}\right)\left\{u_{i_{j}}\left(x_{j}\right)-h\left(x_{j-1}\right) v_{i_{j}}\left(x_{j}\right)\right\},
$$

and since by the first part $x_{j-1}$ is nonoptimal [i.e., $h\left(x_{j-1}\right)>\vartheta(\mathrm{P})$ ], this yields

$$
u_{i_{j}}\left(x_{j}\right)-h\left(x_{j-1}\right) v_{i_{j}}\left(x_{j}\right)<0,
$$

or equivalently

$$
h\left(x_{j}\right)<h\left(x_{j-1}\right) .
$$

Applying again (11) and using the monotonicity of the sequence $h\left(x_{j}\right)$, $j<j^{*} \leq \infty$, the last result follows.

From the above proposition and the definition of $\left(\mathrm{P}_{j+1}\right)$, it follows that the optimization problem $\left(\mathbf{P}_{j+1}\right)$ reduces to

$$
\vartheta\left(\mathbf{P}_{j+1}\right)=\min _{x \in \mathscr{X}} h_{j}(x) \text {. }
$$


Also,

$$
h\left(x_{j}\right)=\max _{i \in I}\left\{u_{i}\left(x_{j}\right) / v_{i}\left(x_{j}\right)\right\},
$$

and by taking $\mu_{j}=h\left(x_{j}\right)$, it follows by (11) that

$$
\vartheta\left(\mathrm{P}_{j+1}\right)=\min _{x \in \mathscr{X}} h_{j}(x)=\left(1 / \delta_{2}\right) F\left(\mu_{j}\right)+\mu_{j},
$$

where $F\left(\mu_{j}\right)$ denotes the optimal value of the parametric problem, with parameter $\mu_{j}$, used in the Dinkelbach-type procedure (Refs. 2 and 11); i.e.,

$$
F\left(\mu_{j}\right):=\min _{x \in \mathscr{C}} \max _{i \in I}\left\{u_{i}(x)-\mu_{j} v_{i}(x)\right\} .
$$

By our cutting plane interpretation, we also obtain immediately that $\left(1 / \delta_{2}\right) F\left(\mu_{j}\right)+\mu_{j}$ is a lower bound on the value of the optimal solution of $(\mathrm{P})$, and so a good stopping rule is given by

$$
F\left(\mu_{j}\right)<\epsilon \delta_{2}, \quad \text { for some } \epsilon>0 .
$$

Observe that this result can also be proved by analyzing the behavior of the function $F$ around the point $\mu=\vartheta(\mathrm{P})$; see Proposition 2.2 of Ref. 2. Moreover, by Lemma 3.2 and by Propositions 2.1 and 2.2, the well-known results (Ref. 2) that the sequence $\mu_{j}, j \geq 0$, is strictly decreasing and that

$$
\lim _{j \uparrow \infty} \mu_{j}=\vartheta(\mathrm{P}), \quad \lim _{j \uparrow \infty} F\left(\mu_{j}\right)=F(\vartheta(\mathrm{P}))=0
$$

are easily recovered.

Finally, by observing that the Dinkelbach-type algorithm for generalized fractional programming can be seen as an approach based on cutting planes in the space $\mathscr{X}$, we refine and extend the remarks regarding classical fractional programming [i.e., $m=1$, given by Sniedovich (Ref. 12)], and thus we show that this Dinkelbach-type approach is classical par excellence.

Boncompte and Martínez-Legaz in Ref. 8 apply the cutting plane algorithm of Plastria to generalized fractional programs. Hence, they approximate

$$
h(x)=\max _{i \in I}\left\{u_{i}(x) / v_{i}(x)\right\}
$$

by a first-order approximation around the point $x$. In order to derive lower subgradients of the function $h$ at $x$, they construct sets which contain and are contained in the lower subgradient set of $h$; see Proposition 2.1 of Ref. 8. Observe that an improvement of a special case of their proposition corresponds to our Lemma 3.1. Moreover, in order to speed up the solution of the subproblems, one of the versions of the cutting plane algorithm tested in Ref. 8 takes into account a method proposed by Plastria in Ref. 13 for dropping cuts generated in previous iterations. 


\section{Nonstandard Class of Generalized Fractional Programming Problems}

Generalized fractional programs are optimization problems dealing with the minimization of the maximum of finitely many ratios over some feasible region (Ref. 2). As a working hypothesis, it is always assumed that the denominators of the ratios of the objective function are positive on the domain considered. In this section, we will consider a nonstandard class of generalized fractional programs for which the positivity assumption is part of the feasible region. The study of these fractional programs is motivated by an allocation model occurring in stochastic queue location theory. For completeness, this model will be explained in detail at the end of this section.

Let us assume, as in the previous section, that $\mathscr{X} \subseteq \mathbb{R}^{n}$ is compact and that $u_{i}, v_{i}: \mathscr{S} \rightarrow \mathbb{R}$ are continuous functions for all $i \in I:=\{1, \ldots, m\}$ on the open set $\mathscr{I}$ containing $\mathscr{X}$. Moreover, the function $h: \mathscr{X} \rightarrow \mathbb{R} \cup\{+\infty\}$ is given by

$$
h(x):=\max _{i \in I}\left\{u_{i}(x) / v_{i}(x)\right\}
$$

and

$$
\mathscr{X}_{c}:=\left\{x \in \mathscr{X} \mid v_{i}(x)>0 \text {, for every } i \in I\right\} .
$$

We will now consider the generalized fractional program given by

$$
\text { (Q) } \inf _{x \in \mathscr{X}_{c}} h(x) \text {, }
$$

under the assumptions that

(a) $u_{i}(x) \geq 0$, for every $x \in \mathscr{X}$ and $i \in I$.

(b) If $u_{i}(x)=0$, for some $x \in \mathscr{X}$ and $i \in I$, then $v_{i}(x)>0$.

For the optimization problem $(\mathrm{Q})$, the feasibility set $\mathscr{X}_{c}$ may be empty. To check whether $\mathscr{X}_{c}$ is nonempty, by the compactness of $\mathscr{X}$ and the continuity of $v_{i}, i \in I$, it is necessary and sufficient that

$$
\max _{x \in \mathscr{X}} \min _{i \in I} v_{i}(x)>0 \text {. }
$$

In the remainder of this section, we will assume that some $\hat{x} \in \mathscr{X}_{\mathrm{c}}$ is known. Since $h(x) \geq 0$ for every $x \in \mathscr{X}_{c}$ by assumption (a), we may assume that $\hat{\mu}:=h(\hat{x})>0$ without loss of generality. Although the set $\mathscr{X}_{c}$ is in general not closed, it will be shown in Lemma 4.1 that $(Q)$ is solvable. It will also be proved in Lemma 4.2 that the associated parametric problem,

$$
\left(\mathrm{Q}_{\mu}\right) \quad F(\mu):=\inf _{x \in \mathscr{X}_{c}}\left\{\max _{i \in I}\left\{u_{i}(x)-\mu v_{i}(x)\right\}\right\},
$$


is solvable for $\vartheta(\mathrm{Q}) \leq \mu$. This implies by Proposition 3.2 of Ref. 2 that the Dinkelbach-type method of Crouzeix, Ferland, and Schaible can be applied to $(\mathrm{Q})$ and has a linear convergence rate. However, since $\mathscr{X}_{c}$ is in general not closed, it might be difficult to solve each subproblem $\left(\mathrm{Q}_{\mu}\right)$ by standard methods. For instance, if $u_{i}, v_{i}, i \in I$, are affine and if $\mathscr{X}$ is defined by linear constraints, the subproblem $\left(\mathrm{Q}_{\mu}\right)$ is no longer a linear programming problem.

Instead of following the above classical approach to solve (Q), we will show that it is also possible to apply the same algorithm to smaller subproblems. In these subproblems, the constraints on the denominator are dropped from the feasible region. In order to prove the first lemma, we introduce the nonempty compact set $\hat{\mathscr{X}} \subseteq \mathbb{R}^{n}$ given by

$$
\hat{\mathscr{X}}:=\left\{x \in \mathscr{X} \mid u_{i}(x)-\hat{\mu} v_{i}(x) \leq 0, \text { for every } i \in I\right\},
$$

and consider the optimization problem

(Q̂) $\inf _{x \in \hat{\mathscr{X}}} h(x)$.

The following result is easy to prove.

Lemma 4.1. The optimal solution sets of $(\mathrm{Q})$ and $(\hat{\mathrm{Q}})$ coincide and are nonempty,

Proof. To prove the first part, it is enough to verify that

$$
\hat{\mathscr{X}}=\left\{x \in \mathscr{X}_{c} \mid h(x) \leq \hat{\mu}\right\} .
$$

Clearly, for some $x \in \mathscr{X}_{c}$ satisfying $h(x) \leq \hat{\mu}$, it follows that $x \in \hat{\mathscr{X}}$. Also, if $x \in \hat{\mathscr{X}}$, we obtain by assumption (a) that $u_{i}(x) \geq 0$. If $u_{i}(x)=0$, this yields by assumption (b) that $v_{i}(x)>0$. Moreover, since $\hat{\mu}>0$, we obtain for $u_{i}(x)>0$ that

$$
v_{i}(x) \geq(1 / \hat{\mu}) u_{i}(x)>0,
$$

and thus $x \in \mathscr{X}_{c}$. Finally, for $x \in \mathscr{X}_{c}$, it follows that

$$
u_{i}(x)-\hat{\mu} v_{i}(x) \leq 0, \quad \text { for every } i \in I,
$$

if and only if $h(x) \leq \hat{\mu}$, and this proves the result.

To prove the second part observe, by the continuity of $h$ on $\left\{x \in \mathscr{S} \mid v_{i}(x)>0, i \in I\right\}$, the compactness of $\hat{\mathscr{X}}$, and $\hat{x} \in \hat{\mathscr{X}}$, that the optimal solution set of $(\hat{Q})$ is nonempty. This implies by the first result that the optimal solution set of $(Q)$ is also nonempty. 
We still have to show that $\left(\mathrm{Q}_{\mu}\right)$ is solvable for $\mu \geq \vartheta(\mathrm{Q})$. This is achieved by showing that the parametric problem

$$
\left(\mathrm{P}_{\mu}\right) \quad G(\mu):=\min _{x \in \mathscr{X}}\left\{\max _{i \in I}\left\{u_{i}(x)-\mu v_{i}(x)\right\}\right\}
$$

has the same set of optimal solutions as $\left(\mathrm{Q}_{\mu}\right)$ for $\mu \geq \vartheta(\mathrm{Q})$.

Lemma 4.2. For every $\mu \geq \vartheta(\mathrm{Q})$, it follows that $x_{\mu}$ is an optimal solution of $\left(\mathrm{P}_{\mu}\right)$ if and only if $x_{\mu}$ is an optimal solution of $\left(\mathrm{Q}_{\mu}\right)$. Moreover, both optimal sets are nonempty.

Proof. From Lemma 4.1, we have that

$$
\hat{\mathscr{X}}=\left\{x \in \mathscr{X}_{c} \mid h(x) \leq \hat{\mu}\right\} .
$$

Hence, by applying Theorem 4.1 of Ref. 2 to (Q̂) and Lemma 4.1, we obtain that $\hat{F}(\vartheta(\mathrm{Q}))=0$ and $\hat{F}$ is decreasing with

$$
\hat{F}(\mu):=\inf _{x \in \hat{\mathscr{X}}}\left\{\max _{i \in I}\left\{u_{i}(x)-\mu v_{i}(x)\right\}\right\},
$$

the objective value of the parametric problem associated with ( $\hat{Q})$. Since $\hat{\mathscr{X}} \subseteq \mathscr{X}$, this implies that

$$
G(\mu) \leq \hat{F}(\mu) \leq 0, \quad \text { for every } \mu \geq \vartheta(\mathrm{Q}) .
$$

If $x_{\mu} \in \mathscr{X}$ is an optimal solution of $\left(\mathrm{P}_{\mu}\right)$, the above inequality yields

$$
u_{i}\left(x_{\mu}\right)-\mu v_{i}\left(x_{\mu}\right) \leq 0,
$$

for every $i \in I$ and $\mu \geq \vartheta(\mathrm{Q}) \geq 0$. Hence, if $u_{i}\left(x_{\mu}\right)>0$, for some $i \in I$, then necessarily $v_{i}\left(x_{\mu}\right)>0$. Also, if $u_{i}\left(x_{\mu}\right)=0$, then by assumption (b) we obtain that $v_{i}\left(x_{\mu}\right)>0$. This shows that $x_{\mu} \in \mathscr{X}_{c}$, and using $\mathscr{X}_{c} \subseteq \mathscr{X}$, it must follow that $x_{\mu}$ is also an optimal solution of $\left(\mathrm{Q}_{\mu}\right)$.

To prove the reverse implication, we first observe that, by the continuity of $u_{i}, v_{i}, i \in I$, and $\mathscr{X}$ compact, the optimization problem $\left(\mathrm{P}_{\mu}\right)$ has an optimal solution $y_{\mu}$. Hence, by the first part $y_{\mu} \in \mathscr{X}_{c}$, and so for $x_{\mu}$ an optimal solution of $\left(\mathrm{Q}_{\mu}\right)$, it must follow that

$$
\max _{i \in I}\left\{u_{i}\left(x_{\mu}\right)-\mu v_{i}\left(x_{\mu}\right)\right\} \leq \max _{i \in I}\left\{u_{i}\left(y_{\mu}\right)-\mu v_{i}\left(y_{\mu}\right)\right\} .
$$

This yields that $x_{\mu}$ is also an optimal solution of $\left(\mathrm{P}_{\mu}\right)$, and hence the lemma is proved. 
By Lemmas 4.1 and 4.2, and by the observations before Lemma 4.1, it is obvious that the Dinkelbach-type algorithm for solving (Q) can be applied in the following way:

Step 1. Choose $x_{0} \leftarrow \hat{x} \in \mathscr{X}_{c}$, and set $\mu_{1} \leftarrow \hat{\mu}=h\left(x_{0}\right)$ and $k \leftarrow 1$.

Step 2. Solve $G\left(\mu_{k}\right)$, and let $x_{k}$ be an associated optimal solution.

Step 3. If $G\left(\mu_{k}\right)<0$, then set $\mu_{k+1} \leftarrow h\left(x_{k}\right), k \leftarrow k+1$, and return to Step 2. Otherwise, stop.

Although the optimization problem (Q) with a noncompact feasible set can be solved directly by the Dinkelbach-type procedure described in Ref. 2 , the above approach simplifies considerably the feasibility set of the subproblems by deleting the nonnegative constraints on the denominators. This observation improves clearly the applicability of the Dinkelbach-type algorithm to this class of problems. Obvious examples are linear generalized fractional programs belonging to this class.

To conclude this section, we consider an allocation problem in location theory satisfying the assumptions of optimization problem $(\mathrm{Q})$. Let

$$
J:=\left\{a_{1}, \ldots, a_{l}\right\} \subseteq \mathbb{R}^{2}
$$

denote the set of $l$ different demand points, and let

$$
I:=\left\{b_{1}, \ldots, b_{m}\right\} \subseteq \mathbb{R}^{2}
$$

be the set of $m$ different locations of identical facilities. Let the sum of the distances from facility $i$ at $b_{i}, i=1, \ldots, m$ to the demand point $j$ at $a_{j}, j=$ $1, \ldots, l$, and from demand point $j$ to facility $i$ be denoted by the positive constant $d_{i j}$. It is assumed that each demand point $j$ generates calls according to a Poisson process $\underline{\mathrm{P}}_{j}(t), t \geq 0$, with rate $\lambda_{j}>0$ and that the Poisson processes $\underline{P}_{1}(t), \ldots, \underline{P}_{l}(t)$ are independent. In the remainder, calls generated by demand point $j$ are called type $j$ calls. If a call from one of the demand points is assigned to facility $i$, it joins a fictitious queue at facility $i$ and waits for service. To keep the model mathematically tractable, it is assumed that each facility has only one server and that the queuing discipline is workconserving and nonpreemptive. This means that the server is not idle if the queue is nonempty, the queuing discipline does not affect either the amount of service time given to a call or the arrival time of any call, and once a service is started to a given call this service will be completed. Well-known examples of work-conserving and nonpreemptive queuing disciplines are FCFS (first come, first served) and random order of service. Once the server starts to serve a given call, this server travels at unit speed from facility $i$ to the demand point which generated this call and returns after servicing to the home facility $i$. Without loss of generality, it is assumed that the onscene and off-scene service times (Ref. 14) can be ignored, and so the total 
service time $s_{i j}$ given to a type $j$ call by the server of facility $i$ equals $d_{i j}$. Since in our optimization problem we like to determine the best random assignment policy of calls to facilities, it is necessary to introduce random policies $x \in \mathbb{R}^{m l}$ consisting of components $x_{i j}, i=1, \ldots, m, j=1, \ldots, l$, with

$$
x_{i j}:=\operatorname{Pr}\{\text { type } j \text { call is assigned to facility } i\} .
$$

Clearly,

$$
0 \leq x_{i j} \leq 1 \text { and } \sum_{i=1}^{m} x_{i j}=1, \quad \text { for every } j=1, \ldots, l .
$$

After having chosen an arbitrary random policy $x$, it follows by the wellknown superposition and decomposition properties of independent Poisson processes (Ref. 15) that the overall arrival process at facility $i$ is again a Poisson process with rate $\sum_{j=1}^{l} \lambda_{j} x_{i j}$. Moreover, the service time distribution $B_{i}(y, x)$ of an arbitrary call assigned to facility $i$ under the random policy $x$ is given by

$$
\begin{aligned}
B_{i}(y, x) & =\sum_{j=1}^{l} B_{i j}(y) \operatorname{Pr}\{\text { arbitrary call is of type } j\} \\
& =\sum_{j=1}^{l} \mathbf{B}_{i j}(y) \lambda_{j} x_{i j}\left(\sum_{k=1}^{l} \lambda_{k} x_{i k}\right)^{-1}
\end{aligned}
$$

with $B_{i j}(y)$ the service time distribution of the service time of a type $j$ call assigned to facility $i$. By our assumption on the service times, it follows that

$$
B_{i j}(y)= \begin{cases}1, & \text { if } y \geq s_{i j}, \\ 0 & \text { if } y<s_{i j} .\end{cases}
$$

From the above observations, it is clear that, for each fixed random policy $x$, the queuing process at facility $i, i=1, \ldots, m$, is a $\mathrm{M} / \mathrm{G} / 1$ queue with a nonpreemptive work-conserving queuing discipline. Denote now by $W_{i}(x)$ the average amount of unfinished work in the steady state (due to the assigned calls) for the server at facility $i$ if the random policy $x$ is used. By a well-known result for arbitrary $M / G / 1$ systems with a work-conserving discipline (Ref. 16), it follows that

$W_{i}(x)$

$$
= \begin{cases}(1 / 2) \sum_{j=1}^{l} \lambda_{j} s_{i j}^{2} x_{i j}\left(1-\sum_{k=1}^{l} \lambda_{k=1} \lambda_{k} s_{i k} x_{i k}\right)^{-1}, & \text { if } 1-\sum_{k=1}^{l} \lambda_{k} s_{i k} x_{i k}>0, \\ +\infty, & \text { otherwise. }\end{cases}
$$


The objective is now to select that random policy which minimizes the maximum of this so-called average work-in-system (Ref. 16) at each facility $i, i=1, \ldots, m$. Introducing the compact set $\mathscr{X} \subseteq \mathbb{R}^{m l}$ of random policies, i.e.,

$$
\mathscr{X}:=\left\{x \in \mathbb{R}^{m l} \mid x_{i j} \geq 0, \sum_{i=1}^{m} x_{i j}=1, \text { for every } j=1, \ldots, l\right\},
$$

this boils down to the generalized linear fractional programming problem

$$
\min _{x \in \mathscr{X}_{c}} \max _{i=1, \ldots, m} W_{i}(x),
$$

with

$$
\mathscr{X}_{c}:=\left\{x \in \mathscr{X} \mid 1-\sum_{j=1}^{l} \lambda_{j} s_{i j} x_{i j}>0, \text { for every } i=1, \ldots, m\right\} .
$$

Clearly, the above optimization problem satisfies the conditions of problem (Q) and was the main motivation for studying these types of problems.

Finally, we like to remark that no computational experiments for the above problem are included in this paper, since the standard procedure which can be applied to this nonstandard problem is already extensively tested (Ref. 17).

\section{References}

1. Plastria, F., Lower Subdifferentiable Functions and Their Minimization by Cutting Planes, Journal of Optimization Theory and Applications, Vol. 46, pp. 37 53, 1985.

2. Crouzeix, J. P., Ferland, J. A., and Schaible, S., An Algorithm for Generalized Fractional Programs, Journal of Optimization Thoery and Applications, Vol. 47, pp. 35-49, 1985.

3. Plastria, F., Continuous Location Problems and Cutting Plane Algorithms, $\mathrm{PhD}$ Thesis, Vrije Universiteit Brussel, 1983.

4. Konnov, I. V., An Application of the Conjugate Subgradient Method for Minimization of Quasiconvex Functions, Journal of Soviet Mathematics, Vol. 45, pp. 1019-1026, 1989.

5. Martínez-Legaz, J. E., On Lower Subdifferentiable Functions, Trends in Mathematical Optimization, Edited by K. H. Hoffman, J. B. Hiriart-Urruty, C. Lemaréchal, and J. Zowe, Birkhäuser Verlag, Basel, Switzerland, Vol. 84, pp. 197-232, 1988.

6. Plastria, F., The Minimization of Lower Subdifferentiable Functions under Nonlinear Constraints: An All-Feasible Cutting Plane Algorithm, Journal of Optimization Theory and Applications, Vol. 57, pp. 463-484, 1988. 
7. Frenk, J. B. G., Gromicho, J., Plastria, F., and Zhang, S., A Deep-Cut Ellipsoid Algorithm and Quasiconvex Programming, Lecture Notes in Economics, Edited by S. Komlósi, T. Rapcsák, and S. Schaible, Springer Verlag, Berlin, Germany, Vol. 405, pp. 62-76, 1994.

8. Boncompte, M., and Martínez-Legaz, J. E., Fractional Programming by Lower Subdifferentiability Techniques, Journal of Optimization Theory and Applications, Vol. 68, pp. 95-116, 1991.

9. Rockafellar, R. T., Convex Analysis, Princeton University Press, Princeton, New Jersey, 1970.

10. Dinkelbach, W., On Nonlinear Fractional Programming, Management Science, Vol. 13, pp. 492-498, 1967.

11. Crouzeix, J. P., and Ferland, J. A., Algorithms for Generalized Fractional Programming, Mathematical Programming, Vol. 52, pp. 191-207, 1991.

12. Sniedovich, M., Fractional Programming Revisited, European Journal of Operational Research, Vol. 33, pp. 334-341, 1988.

13. Plastria, F., Testing Whether a Cutting Plane May Be Dropped, Revue Belge de Statistique, d'Informatique et de Recheerche Opérationnelle, Vol. 22, pp. 1121, 1982.

14. Berman, O., Larson, R. C., and ChiU, S. S., Optimal Server Location on a Network Operating as an $M / G / 1$ Queue, Operations Research, Vol. 33, pp. 746$752,1985$.

15. Cinlar, E., Introduction to Stochastic Processes, Prentice-Hall, Englewood Cliffs, New Jersey, 1975.

16. Heyman, D. P., and Sobel, M. J., Stochastic Models in Operations Research, Vol. 1, McGraw-Hill, New York, New York, 1982.

17. Ferland, J. A., and Potvin, J. Y., Generalized Fractional Programming: Algorithms and Numerical Experimentation, European Journal of Operational Research, Vol. 20, pp. 92-101, 1985. 